Revue des sciences de l'éducation

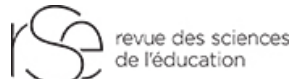

\title{
Caractéristiques individuelles, familiales et scolaires des élèves et leurs influences sur les probabilités de décrochage : analyses selon l'âge du décrochage Individual, family and school characteristics of dropouts according to their age \\ Características individuales, familiares y escolares de alumnos y sus influencias sobre las probabilidades de abandono escolar : análisis según la edad de abandono
}

\section{Aurélie Lecocq, Laurier Fortin et Anne Lessard}

Volume 40, numéro 1, 2014

Texte reçu le 10 septembre 2012, version finale reçue le : 23 mai

2014, accepté le : 6 juin 2014

URI : https://id.erudit.org/iderudit/1027621ar

DOI : https://doi.org/10.7202/1027621ar

Aller au sommaire du numéro

Éditeur(s)

Revue des sciences de l'éducation

ISSN

0318-479X (imprimé)

1705-0065 (numérique)

Découvrir la revue

Citer cet article

Lecocq, A., Fortin, L. \& Lessard, A. (2014). Caractéristiques individuelles, familiales et scolaires des élèves et leurs influences sur les probabilités de décrochage : analyses selon l'âge du décrochage. Revue des sciences de l'éducation, 40(1), 11-37. https://doi.org/10.7202/1027621ar
Résumé de l'article

Nous nous proposons d'étudier les caractéristiques des élèves décrocheurs en fonction de leur âge. Ainsi, nous chercherons (1) à comparer les typologies des élèves décrocheurs de 14 à 17 ans à partir d'analyses descriptives non paramétriques puis (2) à déterminer les probabilités de décrocher en fonction des caractéristiques des élèves selon leur âge, en mobilisant des régressions logistiques binaires. Nos analyses indiquent que les probabilités de décrocher sont associées essentiellement au comportement individuel chez les plus jeunes, et à un ensemble de facteurs scolaires chez les plus âgés. Ainsi, notre étude tend à montrer l'importance de l'âge dans le processus de décrochage scolaire. 


\section{Caractéristiques individuelles, familiales et scolaires des élèves et leurs influences sur les probabilités de décrochage: analyses selon l'âge du décrochage*}

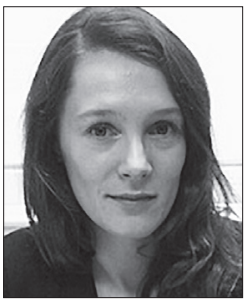

Aurélie Lecocq Consultante en évaluation de programme

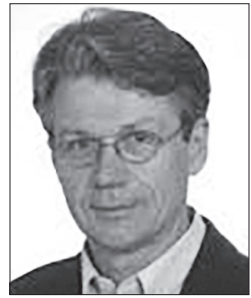

Laurier Fortin

Professeur

Université de Sherbrooke Oghma Consulting

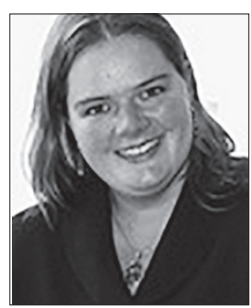

Anne Lessard

Professeure

Université de Sherbrooke

RÉSUMÉ - Nous nous proposons d'étudier les caractéristiques des élèves décrocheurs en fonction de leur âge. Ainsi, nous chercherons (1) à comparer les typologies des élèves décrocheurs de 14 à 17 ans à partir d'analyses descriptives non paramétriques puis (2) à déterminer les probabilités de décrocher en fonction des caractéristiques des élèves selon leur âge, en mobilisant des régressions logistiques binaires. Nos analyses indiquent que les probabilités de décrocher sont associées essentiellement au comportement individuel chez les plus jeunes, et à un ensemble de facteurs scolaires chez les plus âgés. Ainsi, notre étude tend à montrer l'importance de l'âge dans le processus de décrochage scolaire.

MOTS CLÉS (5) • décrochage scolaire, âge, caractéristiques individuelles, caractéristiques familiales et scolaires, typologie.

* Les auteurs remercient la Chaire de recherche de la Commission scolaire de la Région-deSherbrooke sur l'engagement, la persévérance et la réussite des élèves pour son soutien financier à cette recherche postdoctorale. 


\section{Introduction et problématique}

Le décrochage scolaire reste un sujet de préoccupation majeur pour les chercheurs, les décideurs et les intervenants du milieu scolaire, et pour cause: durant l'année scolaire 2010-2011, 16,2\% des jeunes Québécois ont quitté le système scolaire sans diplôme d'études secondaires (ministère de l'Éducation, du Loisir et du Sport, 2012). Un élève est qualifié de décrocheur lorsqu'il quitte le système scolaire sans avoir obtenu son diplôme d'études secondaires et qu'il n'est pas inscrit à l'école l'année suivante (ministère de l'Éducation, du Loisir et du Sport, 2005). Ce taux est sujet à de fortes disparités en fonction des régions (26,6\% pour la commission scolaire de Montréal, 8,3\% pour Baie-James), du genre (23,3\% pour les garçons et $14,3 \%$ pour les filles) et du réseau d'enseignement ( $6,1 \%$ dans le privé contre $18,6 \%$ dans le public) (ministère de l'Éducation, du Loisir et du Sport, 2012) ou de l'âge. En effet, on observe une augmentation progressive du taux de décrochage scolaire en fonction de l'âge de l'élève, particulièrement à partir de 17 ans. En 2000-2001, le décrochage concernait 3,1\% et 5,1\% des élèves de 15 et 16 ans et $11,3 \%$ des élèves de 17 ans. Les données les plus préoccupantes concernent les 5380 jeunes (2,55\%) qui ont décroché avant leur entrée en deuxième secondaire (ministère de l'Éducation, du Loisir et du Sport, 2003). Bien que nous n'ayons pas trouvé d'information dans la recension des écrits concernant l'âge et les caractéristiques de ces décrocheurs précoces, une partie de ces jeunes élèves quitteraient le système éducatif avant 16 ans, l'âge de fin de scolarisation obligatoire. À titre comparatif, le Québec est la province présentant le plus fort taux de décrochage scolaire du Canada (6,2\% en Colombie-Britannique, $7,8 \%$ en Ontario par exemple [Gilmore, 2010]). Les statistiques sur le décrochage scolaire sont souvent difficilement comparables, compte tenu des différences dans la mesure, et dans la définition du décrochage et de la culture. Les statistiques disponibles suggèrent que le Canada, dans son ensemble, a un taux de décrochage scolaire (10,9\%) significativement plus faible que les États-Unis (12,3\%), l'Allemagne (14,2\%) et la France $(14,5 \%)$. Toutefois, certains pays ont un taux plus faible, comme le Royaume-Uni (8\%) et la Norvège (4,6\%) (De Broucker, 2005).

Les conséquences du décrochage sont multiples et affectent autant l'individu que l'ensemble de la société dans laquelle il évolue. Sur le plan individuel, les jeunes qui décrochent vivent des difficultés touchant plusieurs dimensions de leur vie personnelle et professionnelle: la sortie du système éducatif sans diplôme restreint l'intégration socioéconomique; l'accès à l'emploi est plus laborieux et ponctué plus fréquemment de périodes de chômage (Rumberger et Lamb, 2003). Au Canada, le taux de chômage des jeunes de 20 à 24 ans qui ont décroché est de 23,2\%, contre $11,9 \%$ pour les jeunes titulaires d'un diplôme du secondaire, et les décrocheurs ayant un emploi gagnent en moyenne 70 dollars de moins par semaine que leurs homologues diplômés (Gilmore, 2010). En outre, plus le décrochage est précoce, plus il est probable que le jeune rencontrera des difficultés sur le marché du travail (Gilmore, 2010). Le décrochage scolaire est également associé à la délinquance 
juvénile et, adulte, à la consommation excessive d'alcool et de psychotropes ainsi qu'aux comportements à risque pouvant mener à une grossesse précoce (Fortin et Picard, 1999; Janosz, Georges et Parent, 1998; Manlove, 1998). De plus, le décrochage engendre des coûts financiers conséquents pour l'ensemble de la société. Des chercheurs ont estimé le coût total du décrochage scolaire en prenant en compte la réduction des impôts perçus par un salaire moindre et l'augmentation de consommation de programmes sociaux comme les aides sociales, l'assurance chômage et les soins de santé. Levin et Belfield (2007) estiment qu'un décrocheur du secondaire générera une perte économique moyenne de 209000 dollars américains au terme de sa vie active. Cette perte varie de 143000 dollars (pour une femme hispanique) à 268000 dollars (pour un homme noir), selon le genre et l'ethnicité du décrocheur. Carroll et Erkut (2009) ont estimé une perte allant de 89000 dollars (pour un homme blanc) à 201000 dollars (pour un homme noir). Dans le contexte canadien, ce chiffre s'élève en moyenne à 129000 dollars pour un homme et 107000 dollars pour une femme (Lafleur, 1992). Au terme d'une cohorte, on estime que le décrochage scolaire coûte au Québec 1,9 milliard \$, en impôts non perçus et programmes sociaux (Ménard, 2009).

Les conséquences du décrochage sont lourdes et les décrocheurs, nombreux; c'est pourquoi il apparaît nécessaire de mettre en place des stratégies d'intervention. Les programmes de prévention du décrochage scolaire sont d'autant plus efficaces qu'ils sont précoces, et la prévention semble plus apte à maintenir un élève encore scolarisé à l'école que le curatif à l'y faire retourner (Rumberger, 1995). Cependant, pour agir efficacement, il est primordial de comprendre les processus et les mécanismes qui amènent un élève à décrocher, car il s'agit d'un processus complexe et graduel qui se développe par l'interaction de facteurs personnels, familiaux et scolaires (EsterleHedibel, 2006; Fortin, Marcotte, Diallo, Royer et Potvin, 2013; Fortin, Marcotte, Potvin, Royer et Joly, 2006; Robertson et Collerette, 2005). Selon les résultats des quelques études qui ont pris en considération l'âge dans leurs analyses, l'impact de ces facteurs sur les parcours scolaires et le risque de décrochage varie également en fonction de l'âge de l'élève. Afin de cibler plus finement les besoins des élèves en matière de prévention du décrochage, nous nous proposons d'étudier une question essentielle, pourtant peu traitée dans les recherches en sciences de l'éducation: Quelles sont les caractéristiques des élèves qui décrochent selon l'âge et quelles sont les influences de celles-ci sur les probabilités de décrochage? Ainsi, les facteurs personnels, familiaux et scolaires seront présentés brièvement, en indiquant ceux pour lesquels l'âge a été pris en compte lors des analyses.

\section{Recension des écrits : les caractéristiques des élèves à risque de décrochage scolaire}

Les recherches sur le décrochage scolaire peuvent être regroupées en deux grands ensembles: d'une part, une étude de l'impact de facteurs personnels et environnementaux; d'autre part, une analyse par typologie. Si ces deux visions évoluent 
en quasi-autonomie l'une par rapport à l'autre, les combiner peut permettre d'obtenir une vision plus globale et complète du décrochage, choix que nous faisons ici. Nous présentons succinctement notre cadre théorique ainsi que les principaux résultats issus de ces deux approches, d'abord par facteurs, puis par typologie.

Le modèle théorique dans lequel s'ancre cette recherche est le modèle multidimensionnel explicatif du décrochage scolaire développé par Fortin, Marcotte, Diallo, Royer et Potvin (2013). Le décrochage scolaire y est décrit comme la dernière étape d'un processus dynamique et cumulatif de plusieurs facteurs personnels, familiaux et scolaires. Ce modèle, testé auprès de 102 décrocheurs à partir d'équations structurales, présente les facteurs prédictifs du décrochage scolaire. Cinq catégories de facteurs coexistent: deux facteurs familiaux (relation parentsadolescent détériorée, dépression et difficultés familiales), deux facteurs scolaires (climat de classe négatif, interactions à l'école négatives) et un facteur personnel (faible réussite scolaire), mais seule la faible réussite scolaire est en lien direct avec le décrochage scolaire, celle-ci étant influencée par l'ensemble des facteurs.

Sur le plan des caractéristiques personnelles, l'effet du rendement scolaire est particulièrement bien documenté, et il constitue un des plus importants facteurs prédictifs du décrochage (Battin-Pearson, Newcomb, Abbott, Hill, Catalano et Hawkins, 2000; Fortin et al., 2013; Rumberger, 1995, 2011), et le seul facteur en lien direct avec le décrochage scolaire dans le modèle de Fortin et al. (2013). Le genre représente également un facteur incontournable: les garçons ont de plus fortes probabilités de décrocher que les filles, comme l'attestent les statistiques officielles; cependant, le genre perd de sa prédictivité en incluant notamment des variables familiales ou relatives à l'expérience scolaire (Fortin et al., 2013; Janosz, LeBlanc, Boulerice et Tremblay, 1997; Rumberger, 1995). Les facteurs déterminants du décrochage scolaire pour les garçons sont associés à leur comportement à l'école et à la qualité des relations qu'ils développent avec leurs enseignants, leurs pairs, et avec leur famille. Ainsi, il apparaît que les garçons ont généralement plus tendance à décrocher que les filles, car ils ont des aspirations et un engagement scolaire plus faible, ainsi que de moins bonnes relations avec les enseignants (Lessard, Butler-Kisber, Fortin, Marcotte, Potvin et Royer, 2008). Ils participent aussi en classe et ils montrent ou rapportent plus de problèmes de comportements que les filles (Lessard, Fortin, Joly, Royer, Marcotte et Potvin, 2006). Parmi les autres facteurs personnels liés au décrochage scolaire, l'influence de la dépression diffère selon le genre, mais aussi selon l'âge des élèves (Fortin et al., 2006, Marcotte, Fortin, Royer, Potvin et Leclerc, 2001; Quiroga, Janosz et Marcotte, 2006). On observe, chez les filles qui décrochent, une augmentation progressive des symptômes de dépression entre la première et la $5^{\mathrm{e}}$ secondaire, alors qu'ils diminuent avec l'âge chez les garçons (Gagné, Marcotte et Fortin, 2011).

Sur le plan des facteurs familiaux, la communication parent-enfant et l'engagement parental dans la scolarisation de l'enfant contribuent également à expliquer le décrochage scolaire (Dubow, Boxer et Huesmann, 2009; Ou, 2005; Pettit, 
Yu, Dodge et Bates, 2009; Taylor, Hurd, Seltzer, Greenberg et Floyd, 2010). Du point de vue socioéconomique, les enfants provenant de familles monoparentales ou reconstituées, défavorisées ou en dépendance économique et dont les parents ont été peu scolarisés ont une plus grande probabilité de décrocher (Albrecht et Albrecht, 2011; Melby, Conger, Fang, Wickrama et Conger, 2008; Ou, 2005; South, Baumer et Lutz, 2003; Taylor et al., 2010). Outre ces variables, un mauvais encadrement affectif, le manque de pratiques éducatives et de supervision scolaire ainsi que de faibles aspirations scolaires sont autant de facteurs qui peuvent avoir un impact défavorable sur la scolarisation de l'élève.

Sur le plan des facteurs associés à l'école, l'effet de l'assiduité, des comportements inadéquats de l'élève en classe (Garnier, Stein et Jacobs, 1997; Newcomb, Abbott, Catalano, Hawkins, Battin-Pearson et Hill, 2002) et de son engagement scolaire (Archambault, Janosz, Fallu, et Pagani, 2009; Fall et Roberts, 2012; Janosz, Archambault, Morizot et Pagani, 2008, Wang et Fredricks, 2013) sont des facteurs prédictifs de la réussite scolaire et du décrochage. Parmi les facteurs scolaires, certains touchent plus précisément ce qui se passe en classe, comme le climat de classe, l'ordre et l'organisation (Davis et Dupper, 2004; Fortin et al., 2006). La qualité de la relation bilatérale enseignant-élève, que ce soit l'attitude, les attentes et le comportement de l'enseignant envers l'élève ou de l'élève envers l'enseignant, contribue également à l'explication des probabilités de décrochage scolaire (Pianta et Stuhlman, 2004). Des études ont rapporté l'aspect évolutif de certains de ces facteurs, notamment les relations enseignant-élève qui ne sont pas les mêmes en fonction du degré de maturité de l'élève. L'engagement scolaire a longtemps été étudié comme un concept statique et unidimensionnel (Fredricks, Blumenfeld et Paris, 2004), alors qu'il semble évoluer dans le temps (Conseil supérieur de l'éducation, 2008), plus particulièrement pour les élèves à risque de décrochage scolaire, comme le soulignent les travaux de Fortin et al. $(2006 ; 2013)$. Un faible engagement scolaire au début de l'adolescence ou une forte baisse de l'engagement au cours de la scolarité sont fortement prédictifs du décrochage scolaire (Janosz et al., 2008).

Les élèves à risque de décrochage scolaire constituent une population hétérogène aux caractéristiques multiples, ce qui a conduit au développement du deuxième type d'approche, par typologie regroupant les élèves aux caractéristiques semblables. L'analyse de ces typologies permettrait de mieux cerner le caractère évolutif du processus de décrochage scolaire. Les premières typologies de décrocheurs sont théoriques. Elliott et Voss proposent la première dès 1974, et distinguent les décrocheurs handicapés sur le plan intellectuel des élèves capables, mais décrochant à la suite d'expulsions répétitives ou d'ennuis personnels (les involontaires). Kronick et Hargis (1990) proposent un approfondissement, mais obtiennent une typologie des décrocheurs assez similaire. Si l'on ne peut nier l'apport heuristique de ces études, il n'en demeure pas moins que ces typologies n'ont pas reçu de validation empirique. 
Plus récemment, trois études ont cherché à identifier et à valider statistiquement les typologies des élèves décrocheurs (Bowers et Sprott, 2012; Janosz, Le Blanc, Boulerice et Tremblay, 2000), alors que Fortin et al., 2006, ont étudié les élèves à risque de décrochage. Toujours sous un angle empirique, mentionnons l'étude qualitative de Menzer et Hampel (2009) qui distingue les lutteurs, les accablés et les inattendus. Malgré des échantillons différents, les typologies obtenues par Janosz et al. (2000) et Bowers et Sprott (2012) partagent des similarités qui en renforcent la pertinence. Par exemple, Janosz et al. (2000) identifient les discrets ( $40 \%$ de leur échantillon) sortant silencieusement du système là où ce sont les silencieux (52,7\% de l'échantillon) qui sont identifiés par Bowers et Sprott (2012). D’un intérêt particulier pour nous, il est à noter que Bowers et Sprott (2012) relèvent que certains types de décrocheurs sont plus jeunes, même si c'est seulement de quelques mois. Bien que d'un grand intérêt puisqu'elles confirment l'existence de plusieurs profils d'élèves décrocheurs, ces deux études se limitent principalement aux caractéristiques scolaires des élèves.

Fortin et al. (2006) ont développé et validé une typologie d'élèves à risque de décrochage en fonction des facteurs de risque personnels, familiaux et scolaires auprès d'un échantillon de première secondaire. Quatre sous-groupes d'élèves ressortent de leurs analyses: les élèves peu intéressés et peu motivés par l'école $(39,7 \%)$ se caractérisent par un manque de motivation en classe. Ceux rencontrant des problèmes de comportement $(30,5 \%)$ présentent à la fois un faible rendement scolaire et des problèmes de délinquance. Les élèves au comportement antisocial caché (18,9\%) ont généralement recours aux mensonges, au vandalisme, aux vols et aux bagarres. Enfin, les dépressifs (10,7\%) se caractérisent par un score élevé de caractéristiques associées à la dépression des adolescents.

Les caractéristiques des élèves à risque de décrochage sont dans l'ensemble bien documentées et les analyses récentes sur les typologies des décrocheurs fournissent une meilleure compréhension de cette problématique. Bien que le décrochage scolaire soit étudié depuis des décennies, nous n’avons pas recensé d'étude qui traite spécifiquement des caractéristiques des élèves à risque en fonction de leur âge. Pourtant, les études développementales montrent clairement que les jeunes adolescents changent rapidement (Blakemore et Choudhury, 2006) et que les jeunes élèves du premier cycle du secondaire sont différents de ceux du deuxième cycle. Ces connaissances sont essentielles pour développer des programmes de prévention efficaces qui répondent aux besoins des élèves à différents stades de leur scolarité. Ainsi, nous proposons d'étudier le décrochage scolaire en analysant les caractéristiques des élèves qui ont décroché en fonction de leur âge en nous basant sur la typologie qui intègre les trois principaux facteurs de décrochage, celle de Fortin et al. (2006). Nous estimons qu'une meilleure connaissance de ces paramètres permettra une compréhension plus fine du phénomène, une meilleure identification des premières manifestations du processus menant au décrochage scolaire, et ainsi, la mise en place de programmes de prévention 
mieux adaptés. Plus précisément, notre premier objectif est de comparer les types d'élèves de 14 à 17 ans qui ont décroché au moyen d'analyses descriptives non paramétriques. Le second objectif de notre étude est de déterminer l'impact des caractéristiques individuelles, familiales et scolaires des élèves sur les probabilités de décrocher en fonction de leur âge.

\section{Méthodologie}

\subsection{Les participants}

L'échantillon provient d'une étude longitudinale de trois années. Il compte 297 élèves qui ont décroché, scolarisés de la deuxième à la $5^{\mathrm{e}}$ secondaire dans quatre écoles secondaires de la région de l'Estrie. Deux écoles sont situées dans un quartier socioéconomiquement défavorisé et deux dans un quartier de classe sociale favorisée (ministère de l'Éducation, du Loisir et du Sport, 2010). Les élèves ont répondu aux questionnaires alors qu'ils étaient encore à l'école. La typologie des élèves à risque de décrochage a été réalisée à partir du Logiciel de dépistage du risque de décrochage scolaire (Fortin et Potvin, 2007). Comme ils ont quitté l'école sans diplôme et n'y sont pas retournés l'année suivante, ces 297 élèves sont donc considérés comme décrocheurs. Il s’agit majoritairement de garçons (64\%) âgés de 14 à 17 ans (âge moyen: 15,7 ans).

\subsection{Mesures}

Logiciel de dépistage du risque de décrochage scolaire-LDDS (Fortin et Potvin, 2010) Le logiciel permet de dépister les élèves à risque de décrochage scolaire et d'identifier à quel type d'élèves à risque ils appartiennent (Fortin et al., 2006). Le logiciel comprend six questionnaires, dont un qui permet d'identifier le niveau de risque de décrochage et la typologie, et cinq qui permettent d'identifier le type et les caractéristiques personnelles, familiales et scolaires d'élèves à risque. Les qualités psychométriques des questionnaires sont rapportées en détail dans Fortin et Potvin (2010). En ce qui concerne la validité du logiciel, des analyses ont montré que les scores moyens obtenus en lien avec les variables personnelles, familiales et scolaires permettent de différencier de façon significative les élèves à risque et non à risque de décrochage. Enfin, l'expérimentation de ce logiciel auprès de 4500 élèves, chaque année depuis 2007 à ce jour, a permis d'identifier $89 \%$ des élèves qui ont décroché (Fortin et Lessard, 2013).

Questionnaire de dépistage d'élèves à risque de décrochage scolaire Le questionnaire de Potvin, Fortin et Rousseau (2009) a pour objectif d'identifier les élèves à risque de décrochage scolaire au secondaire. Il comprend 39 énoncés, répartis en cinq sous-échelles: Engagement parental (9 items), Attitude envers l'école (9 items), Perception de son niveau de réussite scolaire (10 items), Supervision parentale (7 items) et Aspirations scolaires (4 items). Plus le score obtenu par l'élève est élevé, plus il est à risque de décrochage scolaire. Le questionnaire présente une très bonne 
consistance interne (coefficient alpha de Cronbach de 0,89 ) ainsi qu'une consistance interne acceptable à très bonne pour les sous-échelles, dont les coefficients varient de 0,72 à 0,84 . La fidélité test-retest du questionnaire est supérieure à 0,72 .

\section{Fonctionnement familial}

Ce questionnaire, traduction du Family assessment device (Epstein, Connors et Salinas, 1983), mesure la perception, par l'élève, du fonctionnement familial. Il comprend 60 énoncés, répartis en sept sous-échelles: Résolution de problèmes (6 items), Communication (9 items), Rôles (11 items), Expression affective (6 items), Investissement affectif ( 7 items), Contrôle des comportements (9 items) et Fonctionnement familial global (12 items). L'élève doit répondre en fonction d'une échelle de type Likert en quatre points, de 1 (tout à fait d'accord) à 4 (tout à fait en désaccord). Plus le score obtenu est élevé, plus l'élève est considéré comme à risque. Le questionnaire présente une consistance interne satisfaisante pour six sous-échelles, dont les coefficients varient de 0,66 à 0,88 . Seule la sous-échelle de Résolution de problème présente une valeur inférieure à 0,45 . La fidélité test-retest du questionnaire varie de 0,66 à 0,76 .

\section{Environnement de classe}

Ce questionnaire est une version traduite et abrégée du Classroom environment scale-CES de Moos et Tricket (1987). Il mesure la perception du jeune en ce qui a trait au climat social dans la classe. Le questionnaire comprend neuf souséchelles: Engagement (4 items), Affiliation (4 items), Soutien de l'enseignant (4 items), Orientation vers le travail (4 items), Ordre et organisation (4 items), Clarté des règlements (4 items) et Innovation (4 items). L'élève doit répondre par vrai ou par faux à l'ensemble de ces questions. Plus le score de l'élève est faible, plus ce dernier est à risque. En ce qui concerne la consistance interne, l'étude de Fortin et Potvin (2007) montre que les coefficients alpha de Cronbach varient entre 0,73 et 0,80 pour l'échelle totale et entre 0,46 et 0,65 pour les sous-échelles. Les faibles coefficients associés aux sous-échelles peuvent s'expliquer par le petit nombre d'éléments qui les constitue.

\section{Comportements de l'adolescent}

Traduction du Child behavior checklist-youth self-report (Achenbach, 1991), ce questionnaire mesure les difficultés de comportement de l'élève durant les six derniers mois. Il comprend 113 énoncés, répartis en huit sous-échelles: Anxiétél dépression, Retrait/dépression, Somatisation, Problèmes sociaux, Problèmes de la pensée, Problèmes d'attention, Comportements délinquants et Comportements agressifs. L'élève doit répondre sur une échelle de type Likert en trois points: Jamais, Parfois et Toujours. Plus le score obtenu par l'élève est élevé, plus il présente de problèmes de comportement. La cohérence interne du Child behavior checklistyouth self-report a été évaluée à 0,95 pour les problèmes comportementaux et à 
0,99 pour la compétence sociale. La fidélité test-retest est respectivement de 0,84 et 0,97 pour les problèmes comportementaux et pour la compétence sociale.

\section{Attitudes/perceptions de la qualité de vie à l'école}

Ce questionnaire est une traduction du School life questionnaire-secondary school version (SLQ-ACER, 1978) et a été validé par Girard et Fortin (2006). Cet outil mesure la perception du jeune en ce qui concerne l'école, ses apprentissages et ses aspirations, les enseignants et les pairs. Il évalue les affects positifs (Sentiment de satisfaction générale), les affects négatifs (Aliénation) et les perceptions sur cinq dimensions importantes de la vie à l'école: l'Attitude envers l'enseignant, les Croyances et les opportunités, la Perception des compétences scolaires, le Statut et estime de soi et l'Intégration sociale. L'élève répond au questionnaire en fonction d'une échelle de type Likert en quatre points, de 1 (tout à fait d'accord) à 4 (tout à fait en désaccord). Plus le score obtenu par l'élève est élevé, plus il a des perceptions négatives de l'école. Les résultats de la validation faite par Girard et Fortin (2006) montrent que les coefficients alpha de Cronbach pour la consistance interne sont très bons $(0,83$ à 0,87$)$.

\section{Dépression}

Cet instrument de mesure est une traduction du Center for epidemiological studies depression scale (CES-D) for children de Radloff (1977). Il est composé de 20 questions permettant d'évaluer l'intensité des symptômes affectifs, comportementaux, cognitifs et somatiques de la dépression. Le jeune doit répondre aux différentes questions en choisissant la fréquence de ses symptômes ou comportements au cours des sept derniers jours, les réponses allant de 0 (rarement ou jamais) à 3 (la plupart ou tout le temps). Un score élevé indique une grande détresse psychologique. L'instrument présente une bonne consistance interne, soit une valeur de l'alpha de Cronbach de 0,87, ainsi qu'une stabilité test-retest satisfaisante.

\subsection{Procédures}

L'équipe de recherche a obtenu un certificat de déontologie de l'Université de Sherbrooke pour réaliser cette étude. Elle a ensuite présenté le projet de recherche aux directeurs des établissements et, une fois l'assentiment du conseil d'administration et les autorisations parentales écrites accordées, elle a procédé à l'administration des questionnaires. Les élèves se sont rendus, en début d'année scolaire, dans le laboratoire informatique de l'école pour répondre aux questions du Logiciel de dépistage du risque de décrochage scolaire. Les jeunes ont répondu aux questionnaires sous la supervision d'assistants de recherche de l'équipe formés à la passation des tests en groupe-classe et d'enseignants de l'école. Le temps de passation a varié de 60 à 90 minutes selon les niveaux de lecture et de compréhension des répondants. Les élèves qui n’avaient pas terminé ont pu poursuivre l'évaluation à une autre période. 
L'anonymat et la confidentialité des informations recueillies ont été garantis tant aux établissements qu'aux familles et aux élèves. Après la présentation des objectifs de l'étude à l'ensemble du groupe d'élèves, ceux qui malgré l'autorisation parentale ne désiraient pas participer à cette évaluation ont été dirigés en salle d'étude.

\subsection{Considérations éthiques}

Les participants ont été joints par l'entremise de l'enseignant. Ce dernier a été informé de l'étude par son directeur, puis un des expérimentateurs l'a rencontré afin de lui expliquer les objectifs de l'étude et de solliciter sa participation. Seuls les élèves présentant une déficience intellectuelle ont été exclus. Puis, les parents ou tuteurs légaux ont été approchés afin d'obtenir leur consentement écrit et pour leur garantir la confidentialité des informations recueillies. Les questionnaires ont été administrés en groupe classes à tous les élèves. Des assistants de recherche, formés à la passation des tests, ont été assignés à chacune des écoles.

\subsection{Analyses}

Les analyses ont été effectuées à l'aide de Statistical package for the social sciencesSPSS version 14 pour Windows. Dans un premier temps, les statistiques descriptives présentent la répartition des typologies des élèves à risque de décrochage en fonction de leur âge. En mobilisant des tests de $\chi^{2}$, nous comparons les proportions d'élèves décrocheurs selon l'âge, le genre et la typologie, d'abord sur l'ensemble de l'échantillon, puis en stratifiant l'échantillon par âge. Ces analyses permettent d'explorer la relation entre l'âge des décrocheurs et leur typologie.

Dans un second temps, une série de régressions logistiques binaires permettent d'identifier l'impact des caractéristiques des élèves sur leurs probabilités de décrocher selon leur âge. La variable dépendante est le décrochage constaté (codé 0 pour les non-décrocheurs; 1 pour les décrocheurs). Les variables indépendantes intégrées dans le modèle de régression sont de types personnel, familial et scolaire. Parmi les variables personnelles figurent le genre de l'élève, des indicateurs scolaires (nombres d'absences non motivées, nombre de suspensions, et notes en français), ainsi que le score des quatre variables suivantes: problème d'attention, comportement délinquant, comportement agressif et dépression. Les trois variables familiales incluses dans les analyses sont: contrôle des comportements, résolution de problèmes et fonctionnement familial. Enfin, les variables associées à l'école comprennent les dimensions suivantes: engagement, soutien de l'enseignant, environnement de classe, satisfaction à propos de l'école, attitude envers l'enseignant, croyance en des opportunités et succès. Hormis le genre de l'élève, le nombre d'absences, le nombre de suspensions et les notes de français (divisées en quartile), toutes les autres variables utilisées dans les analyses sont associées à un niveau d'intensité de risque de décrochage, en fonction du score obtenu par l'élève. Il existe trois niveaux de risque pour toutes les dimensions (non à risque, 
à risque léger, à risque modéré), exception faite de la dépression, qui se subdivise en quatre niveaux de risque (un niveau de risque sévère vient s'ajouter aux précédents). Par exemple, le score de comportement agressif (min-max: 0-34) se répartit de la manière suivante: non à risque (score compris entre 0 et 9), à risque léger (10 et 11) et à risque modéré (12 et plus). Dans les régressions, la catégorie de référence utilisée pour les variables indépendantes est non à risque.

\section{Résultats}

L'objectif est, dans un premier temps, d'analyser, selon leur âge, la typologie des élèves à risque et qui ont décroché et, dans un second temps, de mesurer l'effet respectif des facteurs personnels, scolaires et familiaux sur les probabilités de décrocher en fonction de l'âge des élèves.

\subsection{Décrocheurs et typologies selon l'âge}

Les premières analyses descriptives montrent que la répartition du décrochage scolaire constaté diffère selon l'âge. Les résultats au test du $\chi^{2}$ sont significatifs: $\chi^{2}(6, N=8486)=602,02 ; p=0,000(\mathrm{~V}$ de Cramer: 0,27$)$. Aucun élève âgé de 12 ans, de 13 ans ou de 18 ans n'a décroché durant la période de l'étude. Une faible proportion des élèves de 14 et 15 ans $(0,7 \%$ et $4,7 \%)$ ont décroché et ce sont les élèves âgés de 16 et 17 ans qui sont les plus nombreux à quitter l'école: 10,4\% des élèves de 16 ans et $22,3 \%$ des 17 ans finissent par décrocher. Le tableau 1 présente la répartition des élèves décrocheurs et non décrocheurs en fonction de leur âge.

Tableau 1

Distribution des décrocheurs et des non-décrocheurs selon l'âge

\begin{tabular}{lcccccccc}
\hline & 12 ans & 13 ans & 14 ans & 15 ans & 16 ans & 17 ans & 18 ans & Total \\
\hline Non-décrocheurs & 1281 & 1719 & 1909 & 1922 & 1149 & 192 & 17 & 8189 \\
(\% colonne) & $(100 \%)$ & $(100 \%)$ & $(99,3 \%)$ & $(95,3 \%)$ & $(89,6 \%)$ & $(77,7 \%)$ & $(100 \%)$ & $(96,5 \%)$ \\
Décrocheurs & 0 & 0 & 14 & 94 & 134 & 55 & 0 & 297 \\
$(\%$ colonne $)$ & $(0 \%)$ & $(0 \%)$ & $(0,7 \%)$ & $(4,7 \%)$ & $(10,4 \%)$ & $(22,3 \%)$ & $(0 \%)$ & $(3,5 \%)$ \\
Total & 1281 & 1719 & 1923 & 2016 & 1283 & 247 & 17 & 8486 \\
\hline
\end{tabular}

Dans l'ensemble, le décrochage scolaire touche essentiellement les élèves âgés de 16 à 17 ans, et plus particulièrement les garçons. La répartition des décrocheurs selon le genre diffère également: $\chi^{2}(1, n=8486)=24,1 ; p=0,000$, mais l'association entre les deux variables est faible (le coefficient $\varphi$ est égal à 0,05 ). Les garçons sont deux fois plus nombreux que les filles à quitter le système éducatif $(4,5 \%$ contre 2,5\%). Cependant, ils décrochent dans des proportions similaires selon l'âge. En effet, $4,7 \%$ des décrocheurs des deux sexes ont 14 ans, et $30 \%$ des décrocheurs ont 15 ans et elles sont $34,6 \%$ au même âge à quitter le système scolaire. Enfin, $41,1 \%$ des filles qui décrochent ont 16 ans, contre $47,7 \%$ pour les garçons au même âge. 
Nous avons cherché alors à déterminer si le type des élèves décrocheurs était lui aussi lié à l'âge de l'élève. Le graphique ci-dessous présente la répartition des types des élèves décrocheurs selon leur âge.

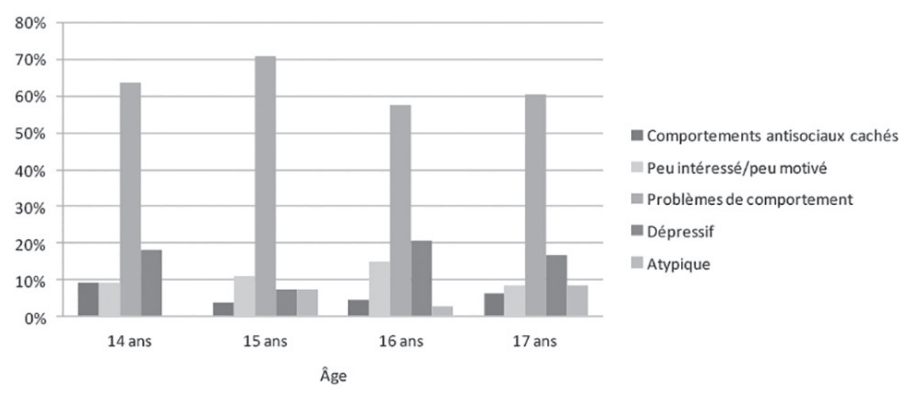

Figure 1. Les typologies des élèves ayant décroché en fonction de leur âge

Les résultats des analyses indiquent que la répartition par types ne varie pas selon l'âge de l'élève ( $\left.\chi^{2}=13,2 ; p=0,349\right)$. Les élèves de 14 ans qui ont décroché rencontrent dans une forte proportion des problèmes de comportement $(63,6 \%)$. Ils sont $18 \%$ à avoir un score élevé de dépression. Enfin, ils sont dans des proportions similaires peu intéressés ou présentent un comportement antisocial caché (9,1\%). Les élèves de 15 ans qui ont décroché rencontrent fréquemment des problèmes de comportement (71,1\%). Ils sont $10,8 \%$ à être peu intéressés, tandis que $7,2 \%$ présentent un score élevé de dépression. Enfin, une faible proportion d'entre eux $(3,6 \%)$ a un comportement antisocial caché. Les élèves de 16 ans rencontrent majoritairement des problèmes de comportement (57,8\%). On constate également qu'une part non négligeable de ces élèves souffre de symptômes dépressifs $(20,7 \%)$ et que $14,7 \%$ sont peu motivés. Enfin, $4,3 \%$ des élèves décrocheurs de 16 ans présentent un comportement antisocial caché. Notons aussi que 2,6\% présentent un profil atypique, c'est-à-dire qu'ils étaient à risque de décrochage, mais qu'ils ne pouvaient être regroupés dans un des quatre types. Une part importante des élèves de 17 ans $(60,4 \%)$ présente des problèmes de comportement. Ils sont ensuite $16,7 \%$ à avoir un score de dépression élevé et $8,3 \%$ à être peu intéressés ou atypiques. Enfin, 6,3\% de ces élèves ont un comportement antisocial caché.

Ainsi, nous constatons que, quel que soit l'âge auquel ils décrochent, une très forte proportion d'élèves rencontre des problèmes de comportement $(57,8 \%$ à $71,1 \%)$. Le pourcentage d'élèves qui décrochent et qui ont un score élevé de dépression varie entre $7,2 \%$ chez les élèves de 15 ans et $20,7 \%$ pour ceux de 16 ans. La proportion d'élèves présentant un comportement antisocial caché reste sous la barre des $10 \%$, quel que soit l'âge. On constate également que le pourcentage d'élèves peu intéressés ou peu motivés demeure relativement constant, à l'excep- 
tion d'une hausse chez les élèves de 16 ans (14,7\%). Enfin, notons que les élèves au profil atypique sont peu nombreux à 16 ans, absents à 14 ans, et représentent $8 \%$ de ceux qui ont décroché à 15 et 17 ans.

Si la typologie des élèves à risque reste stable quel que soit l'âge, elle diffère selon le genre, mais pour les élèves de 15 ans uniquement (les tests du $\chi^{2}$ sont significatifs à $5 \%$ ). On observe un pourcentage plus important de dépression chez les filles que chez les garçons (16,1\% contre 1,9\%). À l'inverse, les garçons sont plus nombreux à rencontrer des problèmes de comportement (80,8\% contre $54,8 \%)$.

\subsection{Impact des caractéristiques des élèves selon l'âge sur les probabilités de décrochage}

Le deuxième objectif est de déterminer si l'impact des caractéristiques des élèves sur les probabilités de décrochage varie en fonction de leur âge. Des analyses préliminaires ont permis de sélectionner un ensemble de variables qui semblent le plus fortement associées au décrochage scolaire: certains items de fonctionnement familial (Family assessment device), d'environnement de classe (Classroom environment scale), de comportement de l'adolescent (Child behavior checklistyouth self-report), de perception de la qualité de vie à l'école (School life questionnaire) et le score de dépression (Center for epidemiological studies scale for children), de même que le genre, les absences et les suspensions et le rendement scolaire. Ces variables sont intégrées aux régressions logistiques binaires dont les résultats figurent dans le tableau 2.

Chez les élèves de 14 ans, nous constatons que le genre ou les absences n'ont pas d'effet significatif sur les probabilités de décrochage, mais un élève qui a été suspendu au cours de l'année est 2,3 fois plus à risque de quitter l'école. L'influence du rendement scolaire sur la probabilité de décrocher est plus forte: un élève du premier quartile a une probabilité 10,9 fois plus élevée de décrocher qu'un élève du premier quartile. Parmi les variables scolaires, l'engagement de l'élève, sa satisfaction et sa perception des compétences scolaires ont un impact significatif sur ces probabilités de décrocher. Parmi les items de fonctionnement familial, la résolution de problèmes a un impact significatif. Enfin, un élève ayant un score élevé de comportement délinquant ou de dépression est 1,4 et 1,6 fois plus à risque de décrocher au cours de l'année.

On observe que toutes les caractéristiques familiales ont un impact significatif sur les probabilités de décrochage chez les élèves de 15 ans, tandis que, à l'inverse, aucun item de l'environnement de classe n'a d'impact significatif. Parmi les autres variables significatives, les absences trop nombreuses, les suspensions, le rendement scolaire, la satisfaction envers l'école et la perception des compétences scolaires contribuent de manière significative aux probabilités de décrochage.

Chez les élèves de 16 ans, peu de variables scolaires, hormis la croyance en des opportunités, ont un impact significatif sur les probabilités de décrochage. Par contre, les élèves présentant un score élevé de problèmes de comportement, de 
Tableau $2 a$

Coefficients de régression logistique binaire de la probabilité de décrocher à 14 ans en fonction des caractéristiques personnelles, scolaires et familiales de l'élève

\begin{tabular}{|c|c|c|c|c|}
\hline Variables indépendantes & & B & S.E & $\operatorname{Exp}(B)$ \\
\hline Constance & & $-2,59$ & 0,18 & $0,07^{* * *}$ \\
\hline \multicolumn{5}{|l|}{ Variables personnelles } \\
\hline Genre (réf: fille) & garçon & $-0,01$ & 0,13 & 0,99 \\
\hline Absences (moins de 5) & 5 et plus & $-0,16$ & 0,16 & 0,85 \\
\hline Suspensions (aucune) & 1 et plus & 0,81 & 0,23 & $2,26^{* * *}$ \\
\hline \multirow[t]{3}{*}{ Français (dernier quartile) } & $1^{\text {er }}$ quartile & 2,39 & 0,21 & $10,95^{* * *}$ \\
\hline & $2^{\mathrm{e}}$ quartile & 1,44 & 0,19 & $4,24^{* * *}$ \\
\hline & $3^{e}$ quartile & 0,71 & 0,2 & $2,03^{* * *}$ \\
\hline Problème d'attention & À risque & $-0,04$ & 0,19 & 0,958 \\
\hline Comportement délinquant & À risque & 0,36 & 0,17 & $1,44^{* *}$ \\
\hline Comportement agressif & À risque & $-0,09$ & 0,21 & 0,91 \\
\hline CES-D dépression & À risque & 0,46 & 0,19 & $1,58^{* *}$ \\
\hline \multicolumn{5}{|l|}{ Variables familiales } \\
\hline Contrôle (non à risque) & À risque & 0,29 & 0,27 & 1,33 \\
\hline Résolution problème & À risque & 0,71 & 0,19 & $2,03^{* * *}$ \\
\hline Fonctionnement familial & À risque & 0,32 & 0,21 & 1,37 \\
\hline \multicolumn{5}{|l|}{ Variables associées à l'école } \\
\hline Engagement & À risque & 0,54 & 0,21 & $1,72^{* * *}$ \\
\hline Soutien de l'enseignant & À risque & $-0,16$ & 1,18 & 0,85 \\
\hline Environnement de classe & À risque & $-0,01$ & 0,26 & 0,99 \\
\hline Satisfaction école & À risque & 1,22 & 0,28 & $3,40^{* * *}$ \\
\hline Attitude envers l'enseignant & À risque & 0,27 & 0,24 & 1,31 \\
\hline Opportunité & À risque & 0,3 & 0,26 & 1,35 \\
\hline Succès & À risque & 0,81 & 0,23 & $2,24^{* * *}$ \\
\hline
\end{tabular}

$R^{2}=0,37$

$N=1761$ (582 décrocheurs et 1179 non-décrocheurs)

Les coefficients sont significatifs au seuil de ${ }^{* * *} p<0,01 ;{ }^{* *} p<0,05 ;{ }^{*} p<0,1$

comportement délinquant ou de comportement agressif sont entre 1,9 et 2 fois plus à risque de décrocher. Il est intéressant de noter que ni le genre ni le score de dépression n'ont d'un impact significatif.

Enfin, les analyses des caractéristiques des élèves décrocheurs de 17 ans montrent que les probabilités de décrochage se polarisent autour des variables ayant trait à l'école. En effet, aucun des coefficients associés aux caractéristiques familiales n'est significatif. Un élève ayant un score de dépression élevé est 25 fois plus à risque de décrocher. Parmi les caractéristiques scolaires, c'est l'environne- 
Tableau $2 b$

Coefficients de régression logistique binaire de la probabilité de décrocher à 15 ans en fonction des caractéristiques personnelles, scolaires et familiales

\begin{tabular}{lllll}
\hline Variables indépendantes & & $\mathrm{B}$ & $\mathrm{S} . \mathrm{E}$ & $\mathrm{Exp}(\mathrm{B})$ \\
\hline Constance & & $-3,077$ & 0,21 & 0,05 \\
Variables personnelles & & & \\
$\quad$ Genre (réf. : fille) & garçon & 0,21 & 0,13 & 1,23 \\
Absences (moins de 5) & 5 et plus & 0,36 & 0,14 & $1,44^{* *}$ \\
Suspensions (aucune) & 1 et plus & 0,45 & 0,23 & $1,56^{*}$ \\
Français (dernier quartile) & $1^{\text {er quartile }}$ & 2,44 & 0,23 & $11,49^{* * *}$ \\
& $2^{\text {d quartile }}$ & 1,69 & 0,21 & $5,41^{* * *}$ \\
& $3^{\text {e quartile }}$ & 0,93 & 0,22 & $2,53^{* * *}$ \\
Problème d'attention & À risque & 0,47 & 0,18 & $1,61^{* * *}$ \\
Comportement délinquant & À risque & 0,38 & 0,16 & $1,46^{* *}$ \\
Comportement agressif & À risque & 0,15 & 0,22 & 1,16 \\
CES-D dépression & À risque & 0,73 & 0,19 & $2,08^{* * *}$ \\
Variables familiales & & & & \\
Contrôle (non à risque) & À risque & 0,89 & 0,26 & $2,443^{* * *}$ \\
Résolution problème & À risque & 0,45 & 0,19 & $1,570^{* * *}$ \\
Fonctionnement familial & À risque & 0,77 & 0,21 & $2,165^{* * *}$ \\
Variables associées à l'école & & & & \\
Engagement & À risque & 0,2 & 0,22 & 1,22 \\
Soutien de l'enseignant & À risque & $-0,01$ & 0,17 & 0,98 \\
Environnement de classe & À risque & $-0,07$ & 0,26 & 0,93 \\
Satisfaction école & À risque & 1,08 & 0,31 & $2,94^{* * *}$ \\
Attitude envers l'enseignant & À risque & $-0,33$ & 0,27 & 0,72 \\
Opportunité & À risque & 0,14 & 0,24 & 1,1 \\
Succès & À risque & 0,87 & 0,24 & $2,39^{* * *}$ \\
\hline & & & &
\end{tabular}

$R^{2}=0,39$

$N=1690$ (565 décrocheurs et 1125 non-décrocheurs)

Les coefficients sont significatifs au seuil de ${ }^{* * *} p<0,01$; ${ }^{* *} p<0,05$; ${ }^{*} p<0,1$

ment de classe qui clairement a le plus d'impact sur le décrochage scolaire. Le rendement scolaire contribue aussi aux probabilités de décrochage. En effet, un élève du premier quartile aura 26 fois plus de risques de décrocher qu'un élève du dernier quartile, et un élève du second quartile aura, quant à lui, sept fois plus de risques de décrocher. Notons enfin qu'un élève qui s'absente plus de cinq fois a six fois plus de risques de décrocher.

Ainsi, les caractéristiques personnelles, familiales et scolaires ne contribuent pas de la même manière aux probabilités de décrochage en fonction de l'âge de 
Tableau 2c

Coefficients de régression logistique binaire de la probabilité de décrocher à 16 ans en fonction des caractéristiques personnelles, scolaires et familiales de l'élève

\begin{tabular}{|c|c|c|c|c|}
\hline Variables indépendantes & & $B$ & S.E & $\operatorname{Exp}(B)$ \\
\hline Constance & & $-3,07$ & 0,26 & $0,05^{* * *}$ \\
\hline \multicolumn{5}{|l|}{ Variables personnelles } \\
\hline Genre (réf. : fille) & garçon & $-0,12$ & 0,18 & 0,88 \\
\hline Absences (moins de 5) & 5 et plus & 0,33 & 0,17 & $1,39^{*}$ \\
\hline Suspensions (aucune) & 1 et plus & 0,23 & 0,28 & 1,26 \\
\hline \multirow[t]{3}{*}{ Français (dernier quartile) } & $1^{\text {er }}$ quartile & 3,01 & 0,29 & $20,36^{* * *}$ \\
\hline & $2^{e}$ quartile & 1,99 & 0,28 & $7,33^{* * *}$ \\
\hline & $3^{e}$ quartile & 0,91 & 0,28 & $2,47^{* * *}$ \\
\hline Problème d'attention & À risque & 0,72 & 0,26 & $2,05^{* * *}$ \\
\hline Comportement délinquant & À risque & 0,62 & 0,2 & $1,86^{* * *}$ \\
\hline Comportement agressif & À risque & 0,62 & 0,2 & $1,88^{* *}$ \\
\hline CES-D dépression & À risque & 0,35 & 0,26 & 1,42 \\
\hline \multicolumn{5}{|l|}{ Variables familiales } \\
\hline Contrôle (non à risque) & À risque & 0,45 & 0,35 & 1,57 \\
\hline Résolution problème & À risque & $-0,19$ & 0,31 & 0,83 \\
\hline Fonctionnement familial & À risque & 1,31 & 0,32 & $3,69^{* * *}$ \\
\hline \multicolumn{5}{|l|}{ Variables associées à l'école } \\
\hline Engagement & À risque & 0,16 & 0,32 & 1,18 \\
\hline Soutien de l'enseignant & À risque & 0,05 & 0,25 & 1,05 \\
\hline Environnement de classe & À risque & 0,27 & 0,35 & 1,3 \\
\hline Satisfaction école & À risque & 0,84 & 0,45 & $2,32^{*}$ \\
\hline Attitude envers l'enseignant & À risque & $-0,71$ & 0,42 & $0,49^{*}$ \\
\hline Opportunité & À risque & 1,06 & 0,3 & $2,89^{* * *}$ \\
\hline Succès & À risque & 0,47 & 0,29 & 1,61 \\
\hline
\end{tabular}

$R^{2}=0,47$

$N=1041$ (390 décrocheurs et 651 non-décrocheurs)

Les coefficients sont significatifs au seuil de ${ }^{* * *} p<0,01 ;{ }^{* *} p<0,05 ;{ }^{*} p<0,1$

l'élève. En ce qui concerne les variables scolaires, on constate que le nombre d'absences injustifiées n'a pas d'impact sur les probabilités de décrochage avant l'âge de 17 ans et que les suspensions ont un impact significatif à l'âge de 14 ans seulement. Si, de manière globale, des variables d'environnement de classe ne contribuent que peu au décrochage scolaire, la satisfaction envers l'école et la perception des compétences scolaires ont un impact significatif chez les élèves jusqu'à 15 ans, et la croyance en des opportunités uniquement chez les élèves de 16 ans. Chez les élèves de 17 ans, l'environnement de classe et la perception des 
Tableau 2d

Coefficients de régression logistique binaire de la probabilité de décrocher à 17 ans en fonction des caractéristiques personnelles, scolaires et familiales de l'élève

\begin{tabular}{|c|c|c|c|c|}
\hline Variables indépendantes & & B & S.E & $\operatorname{Exp}(B)$ \\
\hline Constance & & $-3,07$ & 0,26 & $0,04^{* * *}$ \\
\hline \multicolumn{5}{|l|}{ Variables personnelles } \\
\hline Genre (réf. : fille) & garçon & $-0,62$ & 0,56 & 0,55 \\
\hline Absences (moins de 5) & 5 et plus & 1,93 & 0,59 & $6,89^{* * *}$ \\
\hline Suspensions (aucune) & 1 et plus & $-0,54$ & 0,69 & 0,58 \\
\hline \multirow[t]{3}{*}{ Français (dernier quartile) } & $1^{\text {er }}$ quartile & 3,27 & 1,06 & $26,21^{* * *}$ \\
\hline & $2^{\mathrm{e}}$ quartile & 1,99 & 1,02 & $7,37^{* *}$ \\
\hline & $3^{e}$ quartile & $-0,93$ & 1,38 & 0,39 \\
\hline Problème d'attention & À risque & 0,73 & 1,03 & 2,08 \\
\hline Comportement délinquant & À risque & 0,64 & 0,64 & 1,89 \\
\hline Comportement agressif & À risque & 1,83 & 1,09 & $6,25^{*}$ \\
\hline CES-D dépression & À risque & 3,25 & 1,59 & $25,84^{* *}$ \\
\hline \multicolumn{5}{|l|}{ Variables familiales } \\
\hline Contrôle (non à risque) & À risque & 0,58 & 1,34 & 1,79 \\
\hline Résolution problème & À risque & 2,69 & 1,84 & 14,85 \\
\hline Fonctionnement familial & À risque & $-0,64$ & 1,86 & 0,53 \\
\hline \multicolumn{5}{|l|}{ Variables associées à l'école } \\
\hline Engagement & À risque & $-2,92$ & 2,13 & 0,05 \\
\hline Soutien de l'enseignant & À risque & $-1,71$ & 0,87 & $0,18^{* *}$ \\
\hline Environnement de classe & À risque & 4,42 & 1,75 & $83,19^{* *}$ \\
\hline Satisfaction école & À risque & 0,21 & 0,28 & 1,9 \\
\hline Attitude envers l'enseignant & À risque & $-0,62$ & 1,23 & 0,54 \\
\hline Opportunité & À risque & 0,13 & 1,09 & 1,14 \\
\hline Succès & À risque & 3,28 & 1,53 & $26,59^{* *}$ \\
\hline
\end{tabular}

$R^{2}=0,67$

$N=58$ (73 décrocheurs et 85 non-décrocheurs)

Les coefficients sont significatifs au seuil de ${ }^{* *} p<0,01 ;{ }^{* *} p<0,05 ;{ }^{*} p<0,1$

compétences scolaires sont associés aux probabilités de décrocher. Le rendement scolaire en français est également associé aux probabilités de décrochage, et cela, quel que soit l'âge de l'élève. Cependant, l'ampleur de l'impact diffère considérablement en fonction de l'âge. On observe tout d'abord une hiérarchisation des effets en fonction du niveau de rendement scolaire. En effet, plus un élève a un rendement scolaire faible, plus il est susceptible de décrocher. Cependant, le coefficient associé aux élèves du premier quartile croît considérablement au fur et à mesure que l'élève grandit. $\mathrm{Si}$, à 14 ans, un élève du premier quartile a 10,9 fois 
plus de risques de décrocher, ce rapport de cote atteint 26,2 pour les élèves de 17 ans.

Les caractéristiques personnelles de l'élève contribuent également aux probabilités de décrochage de diverses manières selon l'âge de l'élève. Les problèmes d'attention ont un impact significatif chez les élèves de 15 et 16 ans. Le comportement délinquant a un impact significatif chez les élèves de 14 à 16 ans, mais pas chez les élèves les plus âgés. Dans l'ensemble, c'est lorsque l'élève est âgé de 16 ans que les scores de comportement sont le plus fortement associés aux probabilités de décrochage, alors qu'ils ne le sont plus à 17 ans. Quant au score de dépression, il est associé aux probabilités de décrochage scolaire chez les élèves de 14, 15 ans, et de 17 ans. Le coefficient n'est pas significatif chez les élèves de 16 ans. Enfin, le coefficient associé au genre n'est jamais significatif, quel que soit l'âge de l'élève.

Le dernier ensemble de facteurs contribuant aux probabilités de décrochage est le fonctionnement familial. Il apparaît que le fonctionnement familial n'est pas associé au décrochage scolaire chez les élèves de 17 ans. Par contre, le contrôle a un impact significatif chez les élèves âgés de 15 ans. Enfin, la résolution de problèmes contribue significativement aux probabilités de décrochage chez les élèves de moins de 16 ans et le fonctionnement familial chez les élèves de 15 et 16 ans.

\section{Discussion}

Les recherches antérieures ont cherché à examiner l'impact d'une multitude de variables sur le décrochage scolaire, notamment l'effet des facteurs personnels, familiaux et scolaires des élèves, dont le rendement scolaire, le comportement de l'élève ou son origine sociale par exemple. Si certaines ont porté sur l'étude des disparités selon le genre ou l'ethnicité, aucune à notre connaissance n'a appréhendé les différences afférentes à l'âge de l'élève.

La présente étude visait à examiner l'importance de cette variable selon les caractéristiques et les types d'élèves à risque de décrochage. Dans un premier temps, nous avons observé la répartition des types d'élèves décrocheurs en fonction de l'âge. Cette analyse montre tout d'abord que les décrocheurs sont des adolescents qui rencontrent majoritairement des problèmes de comportement, puisqu'ils représentent $57,8 \%$ à $71,1 \%$ des cas de décrochage avérés. Ces résultats sont congruents avec les conclusions de recherches précédentes identifiant les problèmes de délinquance et d'agressivité comme associés au décrochage scolaire (Blaya, 2010; Fortin et al., 2004). Nos analyses suggèrent également que les profils d'élèves qui décrochent ne diffèrent pas significativement selon l'âge. Ainsi, la typologie des élèves qui ont décroché est sensiblement la même pour les élèves de 14 à 17 ans.

Dans un second temps, nous avons procédé à l'analyse des modèles de régressions logistiques binaires pour connaître l'impact de certaines caractéristiques des élèves sur leur probabilité de décrochage et comparer l'ampleur de cet effet 
en fonction de l'âge de l'élève. Les résultats de ces analyses montrent que, toutes choses étant égales par ailleurs, le genre n'a pas d'impact significatif sur les probabilités de décrochage, et cela, quel que soit l'âge. Des résultats similaires avaient déjà été rapportés dans les écrits de recherche. En effet, nos résultats, comme ceux du modèle théorique de Fortin et al. (2013) et comme l'étude de Janosz et al. (1997), montrent que les garçons sont plus susceptibles de décrocher que les filles, mais le genre perd de sa prédictivité dès lors qu'on le contrôle par un ensemble de variables individuelles, familiales et scolaires. Ces résultats sont très importants, étant donné que plusieurs gestionnaires et professionnels des commissions scolaires choisissent d'intervenir selon le genre pour contrer le décrochage scolaire, ce qui est un choix non fondé. Ces résultats suggèrent plutôt qu'il faut intervenir selon les caractéristiques des élèves, indépendamment du genre. Nous observons néanmoins quelques différences selon le genre chez les élèves de 15 ans. À cet âge, on trouve 8,5 fois plus de filles dépressives que de garçons et, inversement, le taux de garçon avec des problèmes de comportement est supérieur de 47 points de pourcentage à celui des filles. Les recherches en psychologie ont mis en évidence que les filles sont plus susceptibles que les garçons de souffrir de dépression; entre autres, Nolen-Hoeksema (2001) estime qu'elles ont une plus grande sensibilité au stress et de plus fortes probabilités à subir des violences. De la même manière, les garçons sont toujours surreprésentés parmi les élèves qui montrent des problèmes de comportement (Fortin et al., 2004). Nous constatons aussi que l'effet des autres variables individuelles est différent selon l'âge de l'élève.

Chez les élèves les plus jeunes, les probabilités de décrocher sont associées essentiellement aux comportements de l'élève: comportements délinquants, incapacité à résoudre les problèmes avec les parents, suspensions de l'école et insatisfaction envers l'école. Pour les élèves de 15 ans, le comportement délinquant de l'élève s'accompagne de problèmes d'attention. Les probabilités de décrocher sont également associées aux suspensions, ainsi qu'aux nombres d'absences injustifiées. Notons également l'impact des comportements agressifs chez les élèves de 16 ans. Ces résultats rejoignent les conclusions des recherches précédentes plaçant la fréquence élevée des troubles de comportements comme un des facteurs les plus associés au décrochage scolaire (Jimerson, Egeland, Sroufe et Carlson, 2000). Fortin et al. (2004) ont mis en évidence que les élèves ayant un comportement agressif ont un niveau de rendement scolaire très faible. Dans notre étude, nous montrons que ces deux variables jouent un rôle important sur les probabilités de décrocher. Des comportements inappropriés en classe, comme un comportement agressif ou délinquant, peuvent avoir pour conséquence un nombre élevé de suspensions et d'absences. Ces éléments peuvent amener le jeune à se marginaliser encore plus par rapport au milieu scolaire (Blaya, 2003).

Enfin, pour les élèves décrocheurs les plus âgés, c'est essentiellement un ensemble de facteurs scolaires qui affectent les probabilités de décrochage. Outre l'impact du rendement scolaire, qui est plus fort pour les élèves les plus faibles, le 
nombre d'absences injustifiées, l'environnement de classe et les attentes de l'élève envers l'école contribuent fortement à expliquer sa sortie du système scolaire. Notons également le fort impact du score de dépression. Nos résultats vont dans le même sens que le modèle théorique de Fortin et al. (2013) et que ceux de BattinPearson et al. (2000), qui identifient le rendement comme variable centrale dans le processus de décrochage scolaire. Nous apportons toutefois les nuances importantes liées aux différences selon l'âge.

Bien que les résultats de cette recherche fournissent une source d'information pertinente et aident à une meilleure compréhension du décrochage scolaire, quelques limites méritent d'être soulignées. La première a trait à la nature de l'analyse: elle représente une sorte de photographie figée sur un âge donné sans aperçu de trajectoire. Une étude prenant en compte la dimension longitudinale des données ouvrirait alors une perspective instructive afin de pouvoir examiner les changements de typologie ou de statut (non à risque par rapport à risque) au fil des âges, et permettrait d'examiner plus précisément les relations entre l'âge de l'élève et le décrochage. Une seconde limite concerne justement la variable de sélection: l'âge de l'élève. En effet, au même âge, les élèves ne sont pas forcément scolarisés à un niveau scolaire identique, à cause du redoublement par exemple. La dernière limite soulevée concerne les variables introduites dans notre modèle. Deux variables pourraient contribuer à une meilleure compréhension du décrochage scolaire selon l'âge de l'élève: l'ethnicité et l'origine sociale.

\section{Conclusion}

Le décrochage scolaire est étudié depuis des décennies et, pourtant, peu d'études font mention des caractéristiques spécifiques des élèves à risque en fonction des différents âges. Or, il s'agit d'un point essentiel pour développer des programmes de prévention efficaces répondant aux besoins des élèves à différents stades de leur scolarité. En effet, une meilleure connaissance de ces paramètres permettra une compréhension plus fine du phénomène, une meilleure identification des premières manifestations du processus qui mène au décrochage scolaire et, ainsi, la mise en place de programmes de prévention mieux adaptés. Nous avons donc étudié le décrochage scolaire en analysant les caractéristiques des élèves décrocheurs en fonction de leur âge en nous basant sur la typologie de Fortin et al. (2006). Plus précisément, notre premier objectif était de comparer les élèves de 14 à 17 ans qui ont décroché au moyen d'analyses descriptives non paramétriques. Le second objectif de notre étude était de déterminer l'impact des caractéristiques individuelles, familiales et scolaires des élèves sur les probabilités de décrocher en fonction de leur âge.

Ainsi, nos résultats montrent qu'un ensemble de facteurs sont associés aux probabilités de décrocher. Les résultats des régressions logistiques permettent d'identifier les variables qui sont le plus associées au décrochage scolaire selon l'âge. L'âge de l'élève tient une place centrale dans le processus de décrochage scolaire. À des âges différents, ce sont des variables différentes qui auront le plus 
d'impact sur les probabilités de décrochage. Les variables familiales et le score de dépression s'avèrent fortement associés aux parcours des élèves de 14 et 15 ans, comme le suggère aussi le modèle théorique de Fortin et al. (2013). Pour ceux de 15 et 16 ans, c'est avant tout l'impact négatif du comportement qui ressort, tandis que pour les décrocheurs les plus âgés, les facteurs scolaires et la dépression semblent primordiaux. Il est intéressant de noter l'impact croissant du rendement scolaire et l'écart qui se creuse au fur et à mesure entre les élèves les plus faibles et les autres. En effet, les probabilités de décrochage sont hiérarchisées en fonction du niveau de l'élève: un élève du premier décile présente un risque plus élevé de décrocher qu'un élève du second décile, et ainsi de suite. L'écart entre ces élèves s'accroît considérablement en fonction de l'âge de l'élève, ce qui suggère un effet cumulatif des difficultés d'apprentissage de l'élève.

Les résultats ont des implications importantes pour mieux comprendre le processus du décrochage scolaire. Nous avons retenu un ensemble de variables afin de prédire le décrochage scolaire et nous avons combiné cette analyse avec une approche différenciée par types des décrocheurs scolaires. La présente recherche permet de confirmer que c'est la combinaison et le cumul d'un nombre important de facteurs personnels, familiaux et scolaires qui favorise le décrochage scolaire. En effet, ce n'est pas un facteur unique qui explique à lui seul le décrochage scolaire, mais bien un ensemble de variables, différentes selon les élèves, mais aussi selon leur âge. Nous avons montré également que les meilleurs prédicteurs du décrochage varient en fonction de l'âge de l'élève. Un tel résultat signifie que l'âge de l'élève est un élément central dans la compréhension des parcours scolaires. Plutôt que de tenir compte principalement du genre, une piste d'intervention intéressante mérite d'être soulevée. Celle-ci tiendrait compte des besoins spécifiques des élèves en fonction de leur âge et de leur type: il semble essentiel de travailler sur les problèmes de comportements des élèves de 15 et 16 ans, et sur la dépression et les variables scolaires chez les élèves de 17 ans. Les résultats du programme de prévention du décrochage Trait d'union montrent que cette orientation a donné d'excellents résultats (Fortin, 2012; Poirier et Fortin, 2013). De plus, le décrochage scolaire est un processus complexe dont un des facteurs centraux est les difficultés rencontrées à l'école: absences, suspensions, engagement, environnement de classe, mais surtout rendement scolaire. Ces résultats nous amènent à nous interroger sur la place et le rôle de l'école, des enseignants, des directeurs et autres acteurs scolaires dans le processus de décrochage scolaire. Par là-même, ces acteurs peuvent également jouer un rôle central dans la prévention du décrochage et la détection des décrocheurs potentiels. De plus, comme la salle de classe offre un terrain de recherche et d'intervention particulièrement riche, des stratégies d'intervention spécifique portant sur la qualité de la relation élève-enseignant ou entre les élèves pourraient être implantées et évaluées.

En résumé, les résultats générés dans le cadre de cette recherche suscitent des pistes d'études futures prometteuses. Une recherche destinée à examiner les 
trajectoires des typologies des élèves, pour appréhender leur changement de statut (non à risque comparativement à à risque de décrochage) ou de typologie, permettrait une meilleure compréhension des parcours des élèves. Il serait non seulement instructif d'identifier ces différentes trajectoires et de les mettre en relation avec les caractéristiques des élèves, mais aussi de connaître l'impact de celles-ci sur le processus de décrochage scolaire.

\section{English title $\cdot$ Individual, family and school characteristics of dropouts according to their age}

Summary - This study examines the characteristics of students who dropped out of school according to their age. Thus, we seek (1) to compare the typologies of dropouts aged from 14 to 17 with nonparametric analyses and (2) to determine the probabilities of dropping out depending on student characteristics at different ages, with binary logistic regressions. The results of our analysis indicate that the probabilities of dropping out are associated to student behavior for the younger dropouts, and with a set of school factors for older students. Thus, our study demonstrates the importance of age in the dropout process.

Keywords - Dropping out, age, student characteristics, family and school characteristics, typology.

Titulo • Características individuales, familiares y escolares de alumnos y sus influencias sobre las probabilidades de abandono escolar : análisis según la edad de abandono

Resumen - En este artículo proponemos un análisis de las características de los alumnos que abandonan sus estudios en función de su edad. Así, se quiere: (1) comparar las tipologías de alumnos que abandonan los estudios entre los 14 y los 17 años, vía análisis descriptivos no paramétricos; (2) determinar las probabilidades de abandono escolar en función de las características de los alumnos según su edad, movilizando regresiones logísticas binarias. Nuestros análisis indican que las probabilidades de abandono escolar están asociadas esencialmente al comportamiento individual en los alumnos más jóvenes y a un conjunto de factores escolares en los de más edad. Así, nuestro estudio tiende a mostrar la importancia de la edad en el proceso del abandono escolar.

Palabras clave - Abandono escolar, edad, características individuales, características familiares y escolares, tipología.

\section{Références}

Achenbach, T. (1991). Child behavior checklist: manual for the teacher's report form and profile. Burlington, Vermont: University of Vermont.

Albrecht, C. M. and Albrecht, D. E. (2011). Social status, adolescent behavior, and educational attainment. Sociological spectrum, 31(1), 114-137.

Archambault, I., Janosz, M., Fallu, J. S. and Pagani, L. S. (2009). Student engagement and its relationship with early high school dropout. Journal of adolescence, 32(3), 651-670.

Battin-Pearson, S., Newcomb, M., Abbott, R., Hill, K., Catalano, R. and Hawkins, J. (2000). Predictors of early high school dropout: a test of five theories. Journal of educational psychology, 92(3), 568-582. 
Blaya, C., et Hayden, C. (2003). Construction sociale du refus de l'école: processus de non-scolarisation, de déscolarisation et de décrochage scolaire en France et en Angleterre. Rapport de recherche. Paris, France: Direction de la Prospective et du Développement.

Blaya, C. (2010). Décrochages scolaires: l'école en difficulté. Bruxelles, Belgique: De Boeck.

Blakemore, S.-J. and Choudhury, S. (2006). Development of the adolescent brain: implications for executive function and social cognition. Journal of child psychiatry and psychology, 47(3), 296-312.

Bowers, A. J. and Sprott, R. (2012). Examining the multiple trajectories associated with dropping out of high school: a growth mixture model analysis. The journal of educational research, 105(3), 176-195.

Carroll, S. J. and Erkut, E. (2009). The benefits to taxpayers from increases in students' educational attainment. Santa Monica, California: Rand Corporation.

Conseil supérieur de l'éducation (2008). Au collégial - l'engagement de l'étudiant dans son projet de formation: une responsabilité partagée avec les acteurs de son collège. Avis à la ministre de l’Éducation, du Loisir et du Sport. Québec, Québec: Conseil supérieur de l'éducation.

Davis, K. and Dupper, D. (2004). Student-teacher relationships: an overlooked factor in school dropout. Journal of human behavior in the social environment, 9(1-2), 179-193.

De Broucker, P. (2005). Without a paddle: what to do about Canada's young drop-outs. Ottawa, Ontario: Canadian policy research network.

Dubow, E. F., Boxer, P. and Huesmann, L. R. (2009). Long-term effects of parents' education on children's educational and occupational success : mediation by family interactions, child aggression, and teenage aspirations. Merrill-Palmer quarterly (Wayne State University. Press), 55(3), 224.

Elliott, D. and Voss, H. (1974). Delinquency and dropouts. Lexington, Massachusetts: Lexington Books.

Epstein, J., Connors, L. and Salinas, K. (1983). High school and family partnerships: questionnaires for teachers, parents, and students. Baltimore, Maryland: Johns Hopkins University, Center of families, communities, schools, and children's learning.

Esterle-Hedibel, M. (2006). Absentéisme, déscolarisation, décrochage scolaire, les apports des recherches récentes. Déviance et Société, 30(1), 41-65.

Fall, A. M. and Roberts, G. (2012). High school dropouts: interactions between social context, self-perceptions, school engagement, and student dropout. Journal of adolescence, 35(4), 787-798.

Fortin L. (2012). Trait d'union: programme de prévention du décrochage scolaire, Québec, Québec: Centre de transfert pour la réussite éducative du Québec (CTREQ).

Fortin, L. et Lessard, A. (2013). La prédiction du décrochage scolaire dans le secondaire: analyses du cumul des facteurs de risque des décrocheurs. Dans G. Boudesseul (dir.), Du décrochage à la réussite (p. 191-214). France, France: L’Harmattan.

Fortin, L., Marcotte, D., Diallo, T., Potvin, P., Royer, É. and Joly, J. (2013). A multidimensional model of school dropout from an 8-year longitudinal study in a general high school population. European journal of psychology of education, 28(2), 563-583. 
Fortin, L., Marcotte, D., Potvin, P., Royer, E. and Joly, J. (2006). Typology of student at risk of dropping out of school: description by personal, family and school factors. European journal of psychology of education, 21(4), 363-383.

Fortin, L., Marcotte, D., Royer, É. et Potvin, P. (2005). Facteurs personnels, scolaires et familiaux différenciant les garçons en problèmes de comportement du secondaire qui ont décroché ou non de l'école. Nouveaux cahiers de la recherche en éducation, 8(2), 79-88.

Fortin, L. et Picard, Y. (1999). Les élèves à risque de décrochage: facteurs discriminants entre décrocheurs et persévérants. Revue des sciences de l'éducation, 25(2), 359-374.

Fortin, L. et Potvin, P. (2007). Logiciel de dépistage du décrochage scolaire. Québec, Québec: Centre de transfert pour la réussite éducative du Québec (CTREQ).

Fortin, L. et Potvin, P. (2010). Logiciel de dépistage du décrochage scolaire (LDDS). Québec, Québec: Société GRICS.

Fortin, L., Royer, E., Potvin, P., Marcotte, D. et Yergeau, E. (2004). La prédiction du risque de décrochage scolaire au secondaire: facteurs personnels, familiaux et scolaires. Revue canadienne des sciences du comportement, 36(3), 219-231.

Fredricks, J. A., Blumenfeld, P. C. and Paris, A. H. (2004). School engagement: potential of the concept, state of the evidence. Review of educational research, 74(1), 59-109.

Gagné, M-È., Marcotte, D. et Fortin, L. (2011). L'impact de la dépression et de l'expérience scolaire sur le décrochage scolaire des adolescents. Revue canadienne de l'éducation, 34(2), 77-92.

Garnier, H., Stein, J. and Jacobs, J. (1997). The process of dropping out of school: a 19 year perspective. American educational research journal, 34 (2), 395-419.

Gilmore, J. (2010). Tendances du taux de décrochage et des résultats sur le marché du travail des jeunes décrocheurs. Ottawa, Ontario: Statistiques Canada, Division de la statistique du travail.

Girard, F. et Fortin, L. (2006). Traduction et adaptation du School-Life Questionnaire (document non publié). Sherbrooke, Québec: Faculté d'éducation, Université de Sherbrooke.

Janosz, M., Archambault, I., Morizot, J. and Pagani, L. (2008). School engagement trajectories and their differential predictive relations to dropout. Journal of social issues, 64(1), 21-40.

Janosz, M., Georges, P. et Parent, S. (1998). L'environnement socioéducatif à l'école secondaire: un modèle théorique pour guider l'évaluation du milieu. Revue canadienne de psychoéducation, 27(2), 285-306.

Janosz, M., Le Blanc, M., Boulerice, B. and Tremblay, R.E. (2000). Predicting different types of school dropout: a typological approach on two longitudinal samples. Journal of educational psychology, 92(1), 171-190.

Janosz, M., LeBlanc, M., Boulerice, B. and Tremblay, R. (1997). Disentangling the weight of school dropout predictors: a test on two longitudinal samples. Journal of youth and adolescence, 26(6), 733-762.

Jimerson, S. R., Egeland, B., Sroufe, L. A. and Carlson, B. (2000). A prospective longitudinal study of high school droupouts: examining multiple predictors across development. Journal of school psychology, 38(6), 525-549. 
Kronick, R. and Hargis, C. (1990). Dropouts: who drops out and why and the recommended action. Springfield, Illinois, United Kingdom: Charles C. Thomas.

Lafleur, B. (1992). Dropping Out: The Cost to Canada. Report 83-92-E. Ontario, Canada: Publications Information Centre, The Conference Board of Canada.

Lessard, A., Butler-Kisber, L., Fortin, L., Marcotte, D., Potvin, P. and Royer, É. (2008). Shades of disengagement: high school dropouts speak out. Social psychology of education, 11(1), 25-42.

Lessard, A., Fortin, L., Joly, J., Royer, É., Marcotte, D. et Potvin, P. (2006). Les raisons de l'abandon scolaire: différences selon le genre. Revue québécoise de psychologie, 27(1), 135-152.

Levin, H. M. and Belfield, C. R. (2007). Educational interventions to raise high school graduation rates. In C. R. Belfield and H. M. Levin (eds), The price we pay: economic and social consequences of inadequate education (p. 177-199). Washington, District of Columbia: Brookings Institution Press.

Manlove, J. (1998). The influence of high school dropout and school disengagement on the risk of school-age pregnancy. Journal of research on adolescence, 8(2), 187-220.

Marcotte, D., Fortin, L., Royer, É., Potvin, P. et Leclerc, D. (2001). L'influence du style parental, de la dépression et des troubles de comportement sur le risque d'abandon scolaire. Revue des sciences de l'éducation, 27(3), 687-712.

Melby, J. N., Conger, R. D., Fang, S.-A., Wickrama, K. A. S. and Conger, K. J. (2008). Adolescent family experiences and educational attainment during early adulthood. Developmental psychology, 44(6), 1519-1536.

Ménard, L. J. (2009). Au-delà des chiffres... une affaire de cour. Comment le Québec peut se mobiliser contre le décrochage scolaire. Rapport du Groupe de travail sur la persévérance et la réussite scolaire. Québec, Québec: Bibliothèque nationale du Québec. [Récupéré sur le site http://www.reussiteeducativeestrie.ca/dynamiques/Publications/ complet_au_dela_des_chiffres1.pdf]

Menzer, J. D. and Hampel, R. L. (2009). Lost at the last minute. Phi delta kappan, 90(9), 660-664.

Ministère de l'Éducation, du Loisir et du Sport (2003). Abandon scolaire et décrochage: les concepts. Bulletin statistique de l'éducation. 25(1), 1-7.

Ministère de l'Éducation, du Loisir et du Sport (2005). Indicateurs de l'éducation: Édition 2005. Québec, Québec: Gouvernement du Québec.

Ministère de l'Éducation, du Loisir et du Sport (2010). Indicateurs de l'éducation: Édition 2010. Québec, Québec: Gouvernement du Québec.

Ministère de l'Éducation, du Loisir et du Sport (2012). Indicateurs de l'éducation: Édition 2012. Québec, Québec: Gouvernement du Québec.

Moos, R. and Tricket, E. (1987). Classroom environment scale manual. Palo Alto, California: Consulting Psychologists Press.

Newcomb, M. D., Abbott, R. D., Catalano, R. F., Hawkins, J. D., Battin-Pearson, S. and Hill, K. (2002). Mediational and deviance theories of late high school failure: process roles of structural strains, academic competence, and general versus specific problem behavior. Journal of counseling psychology, 49(2), 172-186. 
Nolen-Hoeksema, S. (2001). Gender differences in depression. Current directions in psychological science, 10(5), 173-176.

Ou, S.-R. (2005). Pathways of long-term effects of an early intervention program on educational attainment: findings from the Chicago longitudinal study. Journal of applied developmental psychology, 26(5), 578-611.

Pettit, G. S., Yu, T., Dodge, K. A. and Bates, J. E. (2009). A developmental process analysis of cross-generational continuity in educational attainment. Merrill-Palmer quarterly, 55(3), 250-284.

Pianta, R. C. and Stuhlman, M. W. (2004). Teacher-child relationships and children's success in the first years of school. School psychology review, 33(3), 444-458.

Poirier, M. et Fortin, L. (2013). Évaluation des effets d'un programme de prévention du décrochage scolaire: le programme Trait d'Union. Éducation et formation - e-300, 124-139.

Potvin, P., Fortin, L. et Rousseau, M. (2009). Qualités psychométriques du questionnaire de dépistage des élèves à risque de décrochage scolaire. Revue de psychoéducation, 38(2), 263-278.

Quiroga, C., Janosz, M. et Marcotte, D. (2006). Les sentiments dépressifs à l'adolescence: un facteur de risque différentiel du décrochage scolaire chez les filles et les garçons de milieu défavorisé. Revue de psychoéducation, 35(2), 277-300.

Radloff, L. S. (1977). The CES-D Scale: A self-report depression scale for research in the general population. Applied psychological measurement, 1(3), 385-401.

Robertson, A. et Collerette, P. (2005). L'abandon scolaire au secondaire: prévention et interventions. Revue des sciences de l'éducation, 31(3), 687-707.

Rumberger, R. (1995). Dropping out of middle school: a multilevel analysis of students and schools. American educational research journal, 32(3), 583-625.

Rumberger, R. W. and Lamb, S. (2003). The early employment and further education experiences of high school dropouts: a comparative study of the United-States and Australia. Economics of education review, 22(4), 353-366.

South, S. J., Baumer, E. P. and Lutz, A. (2003). Interpreting community effects on youth educational attainment. Youth and society, 35(1), 3-36.

Taylor, J. L., Hurd, H. D., Seltzer, M. M., Greenberg, J. S. and Floyd, F. J. (2010). Parenting with mild intellectual deficits: Parental expectations and the educational attainment of their children. American journal on intellectual and developmental disabilities, 115(4), 340-354.

Wang, M. T. and Fredricks, J. A. (2013). The reciprocal links between school engagement, youth problem behaviors, and school dropout during adolescence. Child development, 85(2), 722-737. 


\section{Correspondance}

Aurelie.Lecocq@oghma.ca

Laurier.Fortin@USherbrooke.ca

Anne.Lessard@USherbrooke.ca

\section{Contribution des auteurs}

Aurelie Lecocq: $65 \%$

Fortin Laurier : $25 \%$

Anne Lessard: 10\%

Ce texte a été révisé par: Nancy Allen

Texte reçu le 10 septembre 2012

Version finale reçue le: 23 mai 2014

Accepté le: 6 juin 2014 